\title{
Regulation of fatty acid transport and storage: influence of acylation-stimulating protein
}

\author{
Katherine Cianflone and Sabina Paglialunga \\ Centre de Recherche Hôpital Laval, Québec, Canada
}

\begin{abstract}
Postprandial lipemia and fatty acid fluxes occur several times daily, resulting in very efficient absorption of dietary fat and redistribution to various tissues. Absorbed dietary lipids are incorporated into chylomicrons to distribute triglycerides either for storage in adipose tissue or for immediate use in muscle. Commonly, the dietary sources of fat exceed the actual needs and the tissues are faced with dealing with the excess. Under these circumstances, the removal process of dietary triglycerides and fatty acids becomes overloaded, resulting in excessive postprandial lipemia and accumulation of chylomicrons, remnant particles and non-esterified fatty acids. These particles are associated with disruptions in lipoprotein metabolism and changes in inflammatory factors, thus their association with cardiovascular disease, metabolic syndrome and diabetes is not surprising. Dietary factors, not just fat, influence postprandial fluxes. This leads to the question: do we need a standardized fat tolerance test? The recognition of the factors influencing postprandial lipemia and fatty acid uptake and clearance is constantly increasing. Numerous proteins, transporters, enzymes and hormones have been shown to affect fatty acid flux at the level of absorption, peripheral uptake and hepatic remnant clearance. This summary targets fatty acid fluxes, with a focus on acylation-stimulating protein.
\end{abstract}

Keywords: C3adesArg; lipoprotein lipase; postprandial; triglyceride

Abbreviations: apo: apolipoprotein; ASP: acylation-stimulating protein; BAT: brown adipose tissue; CR: chylomicron remnant; FABP: fatty acid binding protein; FATP: fatty acid transport protein; GLP: glucagon-like peptide; HDL: high-density lipoprotein; LDL: low-density lipoprotein; LPL: lipoprotein lipase; LRP: lowdensity lipoprotein receptor-related protein; MTP: microsomal triglyceride transport protein; NEFA: nonesterified fatty acid; SR: scavenger receptor; TG: triglyceride; TNF- $\alpha$ : tumor necrosis factor- $\alpha$; TRL: triglyceride-rich lipoprotein; VLDL: very low-density lipoprotein; VLDLR: very low-density lipoprotein receptor.

\section{Overview of fatty acid fluxes: intestinal absorption, peripheral lipolysis and hepatic remnant clearance}

$\mathrm{F}$ at is absorbed by cells of the small intestine as fatty acids and cholesterol via direct uptake as well as numerous fatty acid transporter proteins. Esterification of the fatty acids to triglycerides (TGs) and of the cholesterol to cholesterol esters leads to subsequent formation of the lipoprotein particle containing an outer core of phospholipids, cholesterol and apoproteins, with an inner core of neutral lipids (primarily TGs with some cholesterol esters). These dietary lipoproteins are transported into the lymphatic system and then released into plasma in the form of nascent chylomicrons. Within the intestinal cells, both fatty acid binding protein-2 (FABP-2) and microsomal triglyceride transfer protein (MTP) are required for the formation of chylomicrons, which contain apolipoprotein B48 (apoB48). Circulating chylomicrons contain numerous other apoproteins, including apoCI, apoCII, apoCIII, apoE and the recently identified apoAV, which influence metabolism of the lipoprotein particles. Various hormones such as glucagon-like peptide (GLP)-1 and -2 can influence this absorption process.

Within the periphery, lipoprotein lipase (LPL) localized to the endothelial cell surface within the capillaries binds to the chylomicrons, hydrolyzing the TGs to release glycerol and fatty acids for uptake and use (energy in the skeletal and cardiac muscle) or storage in adipose tissue. As the chylomicrons release their TGs in the periphery, they shrink and excess surface constituents 
(phospholipids and apoproteins) are transferred back to high-density lipoprotein (HDL). Up to $70-90 \%$ of the TG is hydrolyzed, leaving a chylomicron remnant (CR) particle. LPL is a key gatekeeper of this clearance process, and in the absence of LPL there is no chylomicron hydrolysis. However, in addition to LPL, there are many collateral players that influence the activity of LPL, including very low-density lipoprotein receptor (VLDLR), apoE, apoCII, apoCIII and apoAV. Fatty acids are transported into peripheral tissues (adipose and muscle) via numerous transporters, including CD36, FABPpm and the fatty acid transport protein (FATP) family of transporters (1). Of the massive amounts of fatty acids released by LPL, 36\% escape peripheral adipose and muscle uptake (2). Decreased efficiency of fatty acid uptake into the target tissue results in product inhibition of LPL, and accumulation of chylomicrons and fatty acids in the circulation. Thus, efficient tissue uptake of the fatty acids and re-esterification is essential for continued efficient functioning of LPL. Acylationstimulating protein (ASP) and insulin are two hormones key in maintaining an efficient two-step process.

Both the CR particles and a portion of the fatty acids released by LPL contribute to fatty acid flux to the liver. Within the liver, many cell-surface receptors have been implicated in clearance of CR through interaction with apoE or LPL associated with CR (3). The uptake of CR is also mediated via cell-surface proteoglycans, hepatic lipase, as well as low-density lipoprotein receptor-related protein (LRP) and low-density lipoprotein (LDL) receptor. Other receptors that may be involved include the VLDLR, which binds apoE, and the scavenger receptor (SR)-B1, a multiligand receptor. Recently, studies in ABCA1 knockout mice have implicated this protein in CR metabolism. Finally, many fatty acid transporters are implicated in uptake of nonesterified fatty acids (NEFAs) (1).

The hepatic influx of both CRs and fatty acids impacts subsequent liver very low-density lipoprotein (VLDL) production, as shown by both in vitro and in vivo studies (4). Recent data suggest that hepatic de novo lipogenesis is increased even in the fed state. Using stable isotope tracers in healthy men, spillover of both CRs and NEFAs into the liver stimulates de novo lipogenesis within a few hours following a meal and increases subsequent VLDL production (5).
VLDL particles are secreted by hepatocytes and contain TG, cholesterol ester, phospholipid and apoB100. The more fatty acids available to drive TG synthesis, the larger the VLDL particle (4). The VLDL particles release TGs to peripheral cells through interaction with LPL in capillaries, which breaks down the TGs into fatty acids (as with chylomicrons), with a shift of apoproteins back to HDL. The intermediate-density lipoprotein remnant particles are taken up by the liver $(50 \%)$ or transformed by hepatic lipase into LDL particles containing mainly cholesterol (free and esterified). LDL particles, which contain apoB as their primary apoprotein, can be taken up either by the liver or by peripheral cells through a receptor-mediated process (LDL receptor).

\section{Do we need a standardized oral lipid tolerance test?}

There are many studies evaluating postprandial fatty acid metabolism. Almost every author presents data on standardized meals, with appropriate comparison to control groups. However, between studies, these "standardized" meals vary widely. As summarized in a recent review (6), fat loads vary widely in almost every parameter possible. Fat load tests vary in the total caloric content, ranging from 250 to $2500 \mathrm{kcal}$, from 50 to $100 \%$ fat, 0 to $50 \%$ carbohydrate and 0 to $20 \%$ protein. Further, the meals may be $100 \%$ liquid, $100 \%$ solid or a mixture, with varying caloric density (a consideration in subjects who have undergone gastric bypass, for example).

The percentage of saturated fatty acids, monounsaturated fatty acids and polyunsaturated fatty acids, and the positional distribution within the fats vary. Diets high in safflower oil are primarily polyunsaturated, diets high in olive oil primarily monounsaturated and diets enriched in butter contain mainly saturated fatty acids. In many cases, the fatty acid composition is not calculated or there are insufficient details. Finally, the addition of other components, such as the type of carbohydrate (complex vs sugar) or protein source, can influence postprandial lipemia.

Further, the times when blood samples are drawn and the length of the studies are not necessarily uniform. The quantification of triglyceride-rich lipoproteins (TRLs) can be based on TG, apoB48 or other components of the TRL, and the separation of the particles by ultracentrifugation into large 
TRLs versus small TRLs varies widely. In the past few years, additional methods for evaluation include retinyl palmitate tracers, immunochemical methods for the isolation of remnant lipoproteins (7) and a remnant-like emulsion breath test (8).

In addition to all of the acute components listed above, changes in dietary patterns and exercise before the fatload test may influence both fasting lipoproteins and postprandial fat responses. The chylomicron response to a fat challenge can vary by as much as $50 \%$ according to the fat, carbohydrate and protein content of daily food intake. Chronic consumption of reduced calorie, high-carbohydrate diets can exaggerate plasma TG levels. In nonobese, normolipidemic subjects, standardizing food intake for 3 days reduced the intraindividual variability associated with postprandial chylomicronemia (assessed as apoB48 concentration), but a more prolonged period may be required to achieve the same effect on postprandial TG lipemia (9).

\section{Gatekeepers}

Various dietary factors contribute to changes in overall postprandial fatty acid fluxes (absorption and/or lipolysis and/or hepatic remnant clearance) without the precise mechanisms necessarily being pinpointed. However, several proteins (transporters, enzymes and hormones) influence flux directly and act as gatekeepers of these processes (Fig. 1). While the complexity is increasing, each protein appears to have a specific and individual role in the overall process. Although it is impossible to cover all details, the following section reviews recent developments in these areas, in particular ASP.

\section{Peripheral adipose lipolysis and lipoprotein lipase}

LPL is a glycoprotein enzyme produced primarily by adipose and muscle. Anchored on the luminal surface of capillary endothelial cells, LPL hydrolyzes the TGs in chylomicrons and VLDL, releasing NEFAs, which are stored (adipose) or oxidized (muscle). LPL is key to this process, and in the absence of LPL (as in genetic mutations) there is a complete absence of chylomicron hydrolysis. Although in humans LPL-derived NEFAs form the major pool of storage TGs, in the absence of LPL (genetic mutation) adipose tissue is still maintained through de novo lipogenesis (10). The enzyme LPL and consequently the overall TG storage process are regulated through both the amounts and the activity of the protein, which in turn are modulated via various protein helpers and through hormonal regulation.

Genetic mutations in LPL can produce partial LPL defects and are associated with changes in lipid profile (11). However, other genetic variations, which are common in the population, may also influence lipoprotein metabolism. The LPL variant S447X, present in $18-22 \%$ of individuals, results in alteration of the penultimate amino acid from serine to a stop codon. This mutation is associated with increased LPL mass, but there are conflicting data and interpretations. Evaluation of postprandial fat clearance has demonstrated that this LPL variant is associated with attenuation of apoB48 and TG postprandial increases and increased preheparin LPL mass (12), consistent with a lower risk for cardiovascular disease. Conversely, the HindIII polymorphisms (also indicated as $\mathrm{H} 1 / \mathrm{H} 2$ ), located in the intron between exons 8 and 9 , is associated with delayed postprandial TG clearance and may contribute to familial combined hyperlipidemia (13).

\section{Apoproteins and other cofactors}

Several apoproteins, as well as the recently described VLDL receptor, influence the overall activity of LPL. A substantial body of evidence has accumulated, showing that apoE polymorphisms play a crucial role in the clearance of TRLs (14), and apoCII is well recognized as a cofactor activator of LPL. Variations in plasma apoCIII have been shown to be strong correlates of fasting and postprandial lipemia responses to high monounsaturated fatty acids and high-carbohydrate diets with increased levels of apoCIII associated with delayed postprandial TG clearance (15).

Recently, a new apoprotein (apoAV) was identified by comparative sequencing. Studies in mice demonstrate a marked increase in fasting plasma TGs in apoAV knockout mice, and overexpression of human apoAV in transgenic mice reduced plasma TGs substantially (16), consistent with correlation studies in humans. Several studies have examined the impact of various apoAV polymorphisms on postprandial lipemia. Moreno et al. (17) and Jang et al. (18) demonstrated that carriers of the $-1131 \mathrm{~T} / \mathrm{C}$ allele had significantly increased postprandial lipemia. However, other studies do not support this. Differences in subject characteristics and maintenance diets as well as the total calories and composition of the fat loads probably contributed 


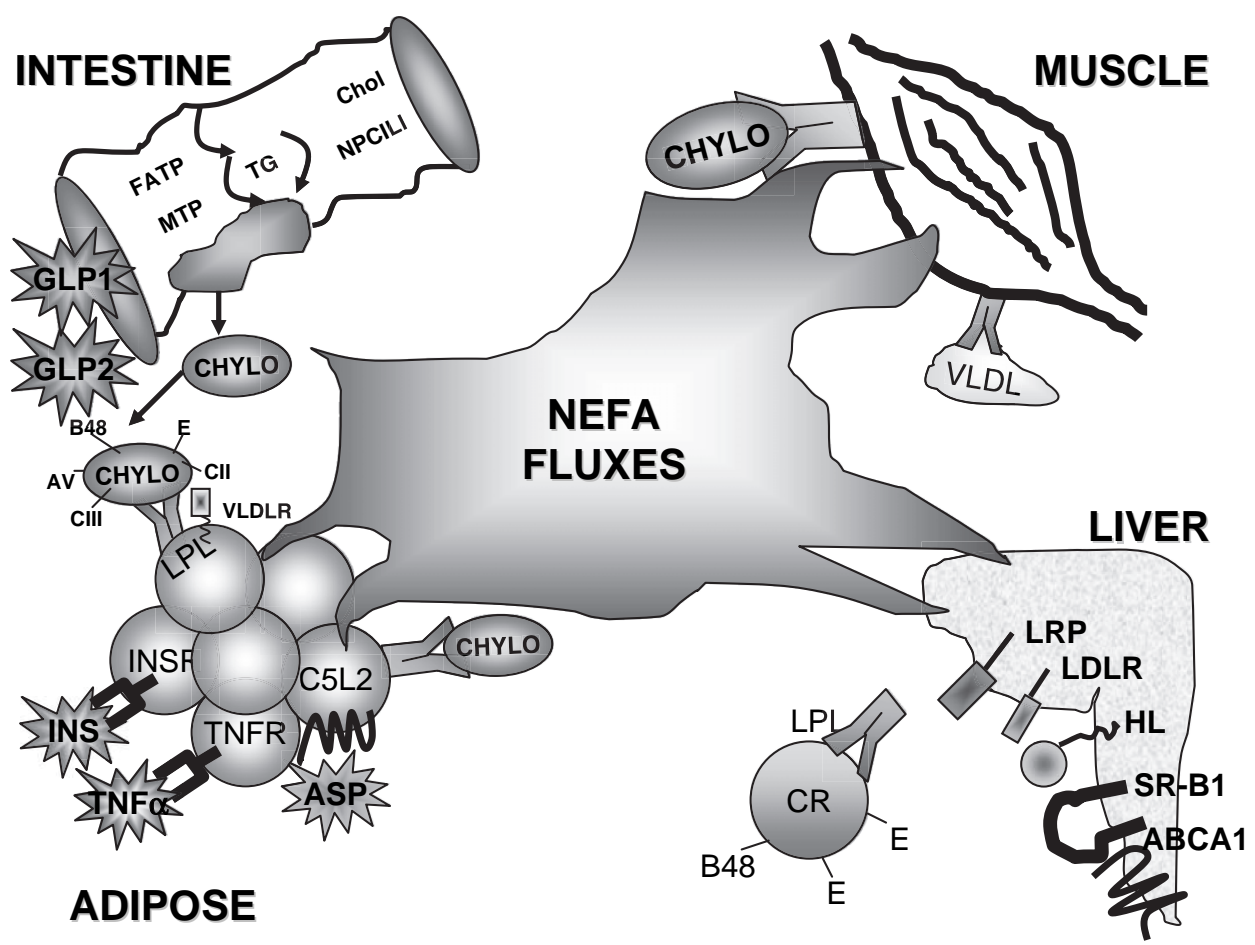

Fig. 1. Determinants of fatty acid fluxes: transporter, enzymes and hormones directly influencing postprandial metabolism, including absorption, lipolysis and hepatic remnant clearance are shown, emphasizing those that play a "gatekeeper" role. Each protein appears to have a specific and individual role in the overall process. ASP: acylation-stimulating protein; Chol: cholesterol; CHYLO: chylomicron; CR: chylomicron remnant; FATP: fatty acid binding protein; GLP: glucagon-like peptide; HL: hepatic lipase; INS: insulin; INSR: insulin receptor; LDL: lowdensity lipoprotein; LDLR: low-density lipoprotein receptor; LPL: lipoprotein lipase; LRP: LDL receptor-like protein; MTP: microsomal triglyceride transport protein; NPC1L1: Niemann-Pick C1-like 1; NEFA: non-esterified fatty acid; TG: triglyceride; TNF $\alpha$ : tumor necrosis factor- $\alpha$; TNFR: tumor necrosis factor receptor; VLDL: very low-density lipoprotein; VLDLR: very low-density lipoprotein receptor.

to these differences. Several mechanisms have been proposed to explain why this polymorphism may be responsible for the differences in postprandial lipemia in humans $(17,18)$ : (i) the $\mathrm{C}$ allele is associated with lower plasma apoAV; (ii) higher levels of apoAV are associated with enhanced LPL activity; (iii) VLDLs lacking apoAV have reduced receptor binding, suggesting reduced remnant clearance; and (iv) apoAV influences the assembly of VLDLs. All of these points are consistent with the decreased VLDL production and increased VLDL catabolism demonstrated in mice.

One additional participant in this lipolytic process is the VLDLR, a member of the LDL receptor family with distinctive ligand-binding properties and tissue distribution. VLDLR is uniquely expressed at sites involved in peripheral TG clearance, including adipose, heart and muscle. VLDLR appears to be involved in the transcytosis of active LPL across endothelial cells, and may facilitate the binding of TG-rich lipoproteins in the capillary bed in concert with LPL (19). The role of VLDLR in chylomicron clearance was evaluated in VLDLR knockout mice. Despite no change in fasting lipid levels, postprandial TG levels were increased ninefold in knockout mice following a fat load. There was no observable effect on adipose tissue uptake of NEFAs, suggesting that VLDLR facilitates postprandial LPL-mediated TG hydrolysis and an absence of VLDLR is associated with reduced activity of LPL (19).

Additional membrane proteins, such as the many fatty acid transporters identified (CD36, FABPpm and the FATP family), which are differentially expressed in various tissues, have also been shown to play an important role in fatty acid uptake and targeting into various lipid pools. Numerous lossof-function and gain-of-function studies in cells and animals have clearly demonstrated that these proteins are important in regulating fatty acid fluxes into and out of cells $(1,20)$.

\section{Hormones and acylation-stimulating protein}

Various hormones contribute to modulation of overall LPL activity. These include insulin, tumor necrosis factor- $\alpha$ (TNF- $\alpha)$, ASP and possibly 
adiponectin. Insulin is well documented as a major LPL modulator. In the postprandial state, adipose tissue LPL is increased relative to fasting levels, whereas muscle LPL tends to be reduced. In adipocytes insulin acutely increases LPL activity and secretion, mostly related to changes at the posttranslational level. This raises the question: are the postprandial increases in LPL solely mediated by the postprandial increases in insulin? Based on studies by Deshaies and coauthors in rodents, the simple answer would appear to be: "yes" (21). Changes in adipose tissue LPL activity were proportional to the changes in insulin, even in the absence of nutrient absorption. Conversely, the usual postprandial changes in adipose and muscle LPL did not occur in the absence of an increase in insulinemia.

Although insulin clearly plays a dominant role in influencing LPL activity, it is not the only component that influences postprandial fat clearance. $\mathrm{TNF}-\alpha$ is a cytokine with a wide range of activities. It is produced primarily by monocytes/macrophages, although significant amounts are secreted by other cell types, including adipocytes, and increased production has been implicated in the pathogenesis of insulin resistance and type 2 diabetes (22). Several variants have been identified that modify gene transcription. TNF- $\alpha$ can potently suppress lipid genes, including LPL and glycerol phosphate dehydrogenase, thereby slowing down not only extracellular lipolysis, but also intracellular processing (22).

It is important to stress that TG clearance occurs as a two-step process: first, the lipoproteins are hydrolyzed by LPL releasing NEFAs; secondly, NEFAs are taken up into the cell and re-esterified to a storage TG molecule. Numerous studies in vitro and in vivo have demonstrated that excess generation of NEFAs by LPL, without prompt and rapid clearance into cells, will result in product inhibition of LPL (23). Thus, processes that amplify the ability of the adipocyte rapidly to take up and store NEFA as TG will indirectly increase the hydrolytic efficiency of LPL. ASP is one such hormone. Also known as C3adesArg, ASP is an adipokine produced through the cleavage of complement $\mathrm{C} 3$ by adipsin (24). ASP interacts with C5L2, a G-protein coupled receptor, activating an intracellular pathway that leads to increased glucose transport and TG storage $(25,26)$. Studies in ASP-deficient, C3 knockout mice demonstrate delayed postprondial TG and NEFA clearance (in spite of normal insulin levels), which are normalized with injection of ASP prior to fotload (24). This effect is maintained in mice treated with a high-fat diet, and in genetically obese $o b / o b$ mice deficient in ASP (double knockout). This delay in TG clearance leads to a leaner mouse with reduced adipose tissue, which is resistant to diet-induced obesity. In vitro studies demonstrated that while ASP has no direct effect on LPL activity (in contrast to insulin), the clearance of TGrich lipoproteins is enhanced by ASP through increasing intracellular TG synthesis, which relieves NEFA inhibition of LPL. The effectiveness of adipose tissue trapping of LPL-derived NEFAs is determined by overall LPL activity, which in turn determines the efficiency of postprandial TG clearance (27). In contrast to the effects of ASP on adipose tissue, ASP decreased in situ muscle LPL activity, similar to the effects of insulin (Fig. 2) (28).

In human studies, fasting ASP is influenced by diet, body size (obesity), exercise and metabolic
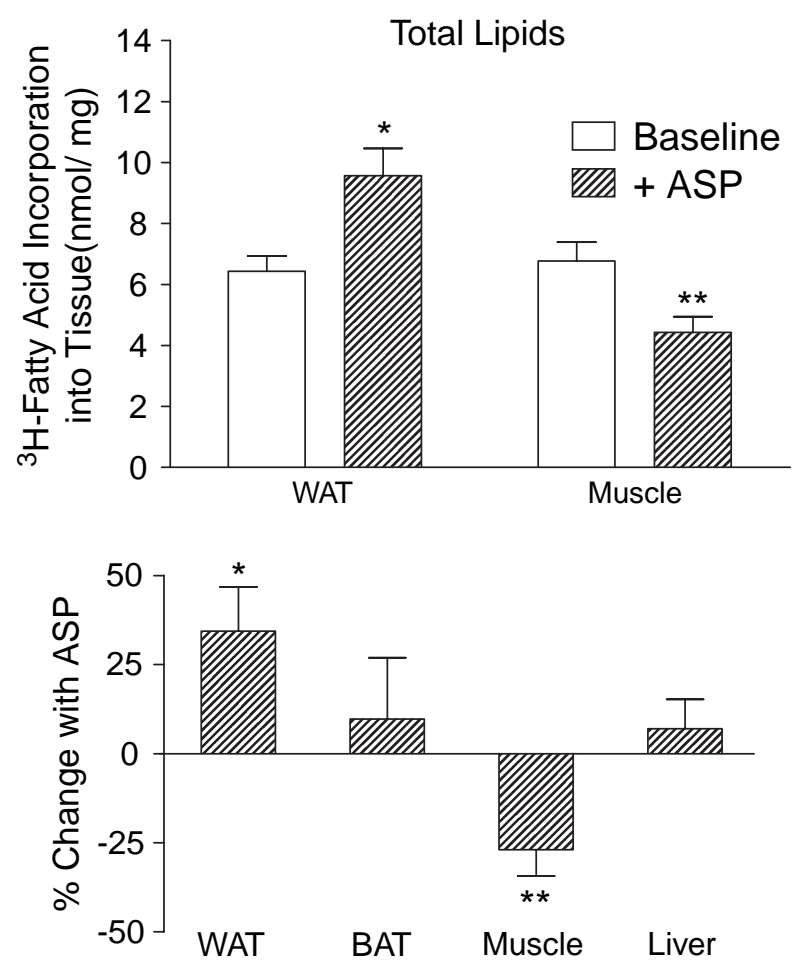

Fig. 2. Acylation-stimulating protein (ASP) increases fatty acid trapping in adipose tissue but decreases in muscle. In situ lipoprotein lipase activity in white adipose tissue (WAT), muscle and brown adipose tissue (BAT). Tissue pieces were incubated for $4 \mathrm{~h}$ with $\left[{ }^{3} \mathrm{H}\right]$ triolein substrate $(1.41 \mathrm{mM})$ with or without acylation-stimulating protein $(1 \mu \mathrm{M})$. Total ${ }^{3} \mathrm{H}$-labeled fatty acid incorporation into total neutral lipids is presented (top panel), with the percentage change without vs with ASP shown in the bottom panel, where $* p<$ 0.05 and ${ }^{* *} p<0.001$ for ASP effect vs baseline, as published previously (28). 
status (presence of cardiovascular disease and/or diabetes) (29-32). In normal healthy men and women, stratification of fasting ASP by tertiles demonstrated a delayed postprandial $\mathrm{TG}$ and NEFA clearance in the subjects with the highest fasting plasma ASP, a correlation that remained even after correction for differences in fasting TG (33). The precursor to ASP, complement C3, also demonstrates these similar associations with TG clearance, as well as correlations with metabolic syndrome indices (34). The association between increased fasting ASP and delayed TG clearance probably reflects ASP resistance, as proposed and described elsewhere (35), similar to the insulin resistance paradigm. Fasting plasma ASP and C3 may be useful markers to identify subjects with postprandial delayed TG and NEFA fluxes, such as those associated with metabolic syndrome.

\section{Summary}

At the level of peripheral clearance, especially in adipose tissue, the central component is LPL. But LPL does not act alone; it is supported by a panoply of stimulatory and inhibitory cofactors (apoCII, apoCIII, apoE, apoAV and LRP) that affect lipolysis either per se or indirectly through adipose tissue trapping of NEFAs. These two processes are influenced at the level of the gene and/or activity by factors such as insulin, TNF- $\alpha$ and ASP and dietary factors, creating a complex web of interdependence.

\section{Acknowledgements}

This study was supported by grants from CIHR and NSERC to KC. KC holds a Canada Research Chair in Adipose Tissue.

\section{References}

1. Hajri T, Abumrad NA. Fatty acid transport across membranes: relevance to nutrition and metabolic pathology. Annu Rev Nutr 2002; 22: 383-415.

2. Miles JM, Park YS, Walewicz D, Russell-Lopez C, Windsor S, Isley WL, et al. Systemic and forearm triglyceride metabolism: fate of lipoprotein lipase-generated glycerol and free fatty acids. Diabetes 2004; 53: 521-7.

3. Van Eck M, Pennings M, Hoekstra M, Out R, Van Berkel TJ. Scavenger receptor BI and ATP-binding cassette transporter A1 in reverse cholesterol transport and atherosclerosis. Curr Opin Lipidol 2005; 16: $307-$ 15 .
4. Saleh J, Sniderman AD, Cianflone K. Regulation of plasma fatty acid metabolism. Clin Chim Acta 1999; 286: $163-80$.

5. Timlin MT, Parks EJ. Temporal pattern of de novo lipogenesis in the postprandial state in healthy men. Am J Clin Nutr 2005; 81: 35-42.

6. Kolovou GD, Anagnostopoulou KK, Daskalopoulou SS, Mikhailidis DP, Cokkinos DV. Clinical relevance of postprandial lipaemia. Curr Med Chem 2005; 12: $1931-$ 45.

7. Cohn JS, Marcoux C, Davignon J. Detection, quantification, and characterization of potentially atherogenic triglyceride-rich remnant lipoproteins. Arterioscler Thromb Vasc Biol 1999; 19: 2474-86.

8. Redgrave TG, Watts GF, Martins IJ, Barrett PH, Mamo JC, Dimmitt SB, Marais AD. Chylomicron remnant metabolism in familial dyslipidemias studied with a remnant-like emulsion breath test. J Lipid Res 2001; 42: 710-5.

9. Slivkoff-Clark K, James AP, Kerr D, Soares MJ, Mamo JC. The effect of diet standardisation on postprandial chylomicron response. Asia Pac J Clin Nutr 2004; 13(Suppl): S69.

10. Brun LD, Gagne C, Julien P, Tremblay A, Moorjani S, Bouchard C, Lupien PJ. Familial lipoprotein lipaseactivity deficiency: study of total body fatness and subcutaneous fat tissue distribution. Metabolism 1989; 38: $1005-9$.

11. Mailly F, Tugrul Y, Reymer PW, Bruin T, Seed M, Groenemeyer BF, et al. A common variant in the gene for lipoprotein lipase (Asp $\rightarrow$ Asn). Functional implications and prevalence in normal and hyperlipidemic subjects. Arterioscler Thromb Vasc Biol 1995; 15: $468-78$.

12. Nierman MC, Rip J, Kuivenhoven JA, van Raalte DH, Hutten BA, Sakai N, et al. Carriers of the frequent lipoprotein lipase S447X variant exhibit enhanced postprandial apoprotein B-48 clearance. Metabolism 2005; 54: 1499-503.

13. Lopez-Miranda J, Cruz G, Gomez P, Marin C, Paz E, Perez-Martinez $\mathrm{P}$, et al. The influence of lipoprotein lipase gene variation on postprandial lipoprotein metabolism. J Clin Endocrinol Metab 2004; 89: 4721-8.

14. Dart A, Sherrard B, Simpson H. Influence of apo E phenotype on postprandial triglyceride and glucose responses in subjects with and without coronary heart disease. Atherosclerosis 1997; 130: 161-70.

15. Archer WR, Desroches S, Lamarche B, Deriaz O, Landry N, Fontaine-Bisson B, et al. Variations in plasma apolipoprotein C-III levels are strong correlates of the triglyceride response to a high-monounsaturated fatty acid diet and a high-carbohydrate diet. Metabolism 2005; 54: 1390-7.

16. Baroukh N, Bauge E, Akiyama J, Chang J, Afzal V, Fruchart JC, et al. Analysis of apolipoprotein A5, C3, and plasma triglyceride concentrations in genetically engineered mice. Arterioscler Thromb Vasc Biol 2004; 24: $1297-302$.

17. Moreno R, Perez-Jimenez F, Marin C, Moreno JA, Gomez P, Bellido C, et al. A single nucleotide 
polymorphism of the apolipoprotein A-V gene $-1131 \mathrm{~T}>\mathrm{C}$ modulates postprandial lipoprotein metabolism. Atherosclerosis 2006; 189: 163-168.

18. Jang Y, Kim JY, Kim OY, Lee JE, Cho H, Ordovas JM, et al. The $-1131 \mathrm{~T} \rightarrow \mathrm{C}$ polymorphism in the apolipoprotein A5 gene is associated with postprandial hypertriacylglycerolemia; elevated small, dense LDL concentrations; and oxidative stress in nonobese Korean men. Am J Clin Nutr 2004; 80: 832-40.

19. Goudriaan JR, Espirito Santo SM, Voshol PJ, Teusink $\mathrm{B}$, van Dijk KW, van Vlijmen BJ, et al. The VLDL receptor plays a major role in chylomicron metabolism by enhancing LPL-mediated triglyceride hydrolysis. J Lipid Res 2004; 45: 1475-81 [Epub 16 May 2004].

20. Kalant D, Cianflone K. Regulation of fatty acid transport. Curr Opin Lipidol 2004; 15: 309-14.

21. Picard F, Naimi N, Richard D, Deshaies Y. Response of adipose tissue lipoprotein lipase to the cephalic phase of insulin secretion. Diabetes 1999; 48: 452-9.

22. Faraj M, Lu HL, Cianflone K. Diabetes, lipids, and adipocyte secretagogues. Biochem Cell Biol 2004; 82: 170-90.

23. Saxena U, Witte LD, Goldberg IJ. Release of endothelial cell lipoprotein lipase by plasma lipoproteins and free fatty acids. J Biol Chem 1989; 264: 4349-55.

24. Maslowska M, Wang HW, Cianflone K. Novel roles for acylation stimulating protein/C3adesArg: a review of recent in vitro and in vivo evidence. Vitam Horm 2005; 70: 309-32.

25. Kalant D, MacLaren R, Cui W, Samanta R, Monk PN, Laporte SA, et al. C5L2 is a functional receptor for acylation-stimulating protein. J Biol Chem 2005; 280: 23936-44.

26. Maslowska M, Legakis H, Assadi F, Cianflone K. Targeting the signaling pathway of acylation stimulating protein. J Lipid Res 2006; 47: 643-52.

27. Faraj M, Sniderman AD, Cianflone K. ASP enhances in situ lipoprotein lipase activity by increasing fatty acid trapping in adipocytes. J Lipid Res 2004; 45: 657-66.

28. Faraj M, Cianflone K. Differential regulation of fatty acid trapping in mouse adipose tissue and muscle by
ASP. Am J Physiol Endocrinol Metab 2004; 287: E1509.

29. Matthan NR, Cianflone K, Lichtenstein AH, Ausman LM, Jauhiainen M, Jones PJ. Hydrogenated fat consumption affects acylation-stimulating protein levels and cholesterol esterification rates in moderately hypercholesterolemic women. J Lipid Res 2001; 42: 1841-8.

30. Schrauwen P, Hesselink MK, Jain M, Cianflone K. Acylation-stimulating protein: effect of acute exercise and endurance training. Int J Obes (Lond) 2005; 29: 632-8.

31. Maslowska M, Vu H, Phelis S, Sniderman AD, Rhode BM, Blank D, et al. Plasma acylation stimulating protein, adipsin and lipids in non-obese and obese populations. Eur J Clin Invest 1999; 29: 679-86.

32. Cianflone K, Xia Z, Chen LY. Critical review of acylation-stimulating protein physiology in humans and rodents. Biochim Biophys Acta 2003; 1609: 127-43.

33. Cianflone K, Zakarian R, Couillard C, Delplanque B, Despres JP, Sniderman A. Fasting acylation-stimulating protein is predictive of postprandial triglyceride clearance. J Lipid Res 2004; 45: 124-31 [Epub 16 October 2003].

34. van Oostrom AJ, Alipour A, Plokker TW, Sniderman $\mathrm{AD}$, Cabezas MC. The metabolic syndrome in relation to complement component 3 and postprandial lipemia in patients from an outpatient lipid clinic and healthy volunteers. Atherosclerosis [Epub ahead of print 17 February 2006].

35. Havel PJ. Update on adipocyte hormones: regulation of energy balance and carbohydrate/lipid metabolism. Diabetes 2004; 53(Suppl 1): S143-51.

\section{Katherine Cianflone}

Centre de Recherche Hôpital Laval Y2 186, 2725 Chemin Ste Foy Québec Canada GIV 4G5

Tel: + | 418656 87| I, ext. 373|

Fax: + | 4186564602

E-mail: katherine.cianflone@crhl.ulaval.ca 